\title{
Strates
}

STRATES Matériaux pour la recherche en sciences sociales

13 | 2007

Paysage urbain: genèse, représentations, enjeux contemporains

\section{Pratiques et compétences paysagistes dans les grands ensembles d'habitation, 1945-1975}

Bernadette Blanchon-Caillot

\section{(2) OpenEdition}

Journals

Édition électronique

URL : http://journals.openedition.org/strates/5723

DOI : $10.4000 /$ strates.5723

ISSN : $1777-5442$

Éditeur

Laboratoire Ladyss

Édition imprimée

Date de publication : 31 décembre 2007

ISSN : 0768-8067

Référence électronique

Bernadette Blanchon-Caillot, « Pratiques et compétences paysagistes dans les grands ensembles d'habitation, 1945-1975 », Strates [En ligne], 13 | 2007, mis en ligne le 05 novembre 2008, consulté le 08 septembre 2020. URL : http://journals.openedition.org/strates/5723 ; DOI : https://doi.org/ $10.4000 /$ strates. 5723

Ce document a été généré automatiquement le 8 septembre 2020.

Tous droits réservés 


\title{
Pratiques et compétences paysagistes dans les grands ensembles d'habitation, 1945-1975
}

\author{
Bernadette Blanchon-Caillot
}

1 Nous souhaitons, dans cet article, éclairer la compréhension du rôle des paysagistes après la Seconde Guerre mondiale, notamment leur participation à la fabrication du paysage urbain dans les grands ensembles d'habitation. L'examen de quelques réalisations choisies montre leur contribution à la constitution d'un nouveau métier. Cet épisode reste peu connu, malgré le développement récent des recherches sur les productions de l'après-guerre. Nous nous intéresserons ici à la production d'une génération de professionnels de l'aménagement, chargés de la conception des espaces ouverts urbains, actifs durant la période dite des Trente Glorieuses (1945-1975) ${ }^{1}$, porteurs d'un héritage lié à la pratique des parcs et jardins et confrontés à une nouvelle échelle de projet sous l'impulsion des mutations profondes de la société.

2 Comprendre les modalités de prise en compte de la dimension du paysage et la façon dont les projets d'aménagement des espaces extérieurs étaient conçus à leur création peut aussi contribuer à nourrir la question de leur actualité, en tant que patrimoine à gérer ou réhabiliter. À travers l'étude de réalisations, il nous paraît important de comprendre dans quelle mesure l'approche spécifique offerte par la compétence paysagiste a alors permis une résolution originale des problématiques urbaines, notamment à l'échelle du territoire ou dans les tissus des grands ensembles.

3 Notre propos s'appuie sur les travaux amorcés dans le cadre du programme de recherche « Architecture des espaces publics modernes » qui portait sur les réalisations de l'après Seconde Guerre mondiale dans leur dimension spatiale comme dans leurs fondements théoriques ${ }^{2}$. L'approche croisée offerte par le séminaire " Paysage urbain » a permis de faire fructifier cette réflexion, mais nous renvoyons à nos précédentes publications ${ }^{3}$ pour compléter cet exposé.

4 Si les paysagistes ont acquis aujourd'hui une certaine légitimité dans la pratique du projet d'aménagement urbain, ils se sont peu mobilisés pour exprimer les fondements 
et enjeux de leur démarche. Et si, depuis une vingtaine d'années en Europe, les réalisations des paysagistes français en milieu urbain sont connues et reconnues, la définition de leur apport spécifique reste peu explicitée et la genèse de leur émergence peu explorée.

5 En outre, il semble que les paysagistes se soient peu impliqués dans la réflexion sur les espaces modernes, le Brésilien Roberto Burle-Marxe étant le seul à participer aux congrès internationaux d'architecture moderne (CIAM) ${ }^{4}$. Ils n'avaient, à la différence des architectes, aucune pratique de l'espace ouvert moderne. Ces derniers, en revanche, déjà actifs dans les débats sur la ville, mobilisés dans l'entre-deux-guerres par les thèses corbuséennes, bénéficient d'une base de réflexion et de savoir-faire plus avancée.

6 Le décalage d'alors, le défaut de prise de distance aujourd'hui, le faible poids des travaux de recherche, ajoutent à l'opportunité qu'il y a à s'intéresser aux œuvres paysagistes, à chercher à y décrypter un positionnement théorique qui n'a pas été formulé. À travers l'examen d'une dizaine d'exemples, nous tenterons d'apporter des éléments de réponse aux questions suivantes; comment ces aménagements ont-ils été pensés et mis en œuvre, quelles compétences engageaient-ils? Quels en étaient les enjeux, à quelles attentes répondaient-ils? Quels enseignements peut-on en tirer? Quels ont été le rôle et le point de vue des différents acteurs?

7 L'exploration du travail paysagiste, dans la période étudiée où se confrontent la dimension $\mathrm{du}$ site et la pensée moderne, nous paraît particulièrement riche à interroger.

8 Nous décrirons ces exemples à travers les savoir-faire utilisés en examinant la commande et son interprétation, la prise en compte du site, la forme des espaces ouverts résultant de l'implantation du bâti, le travail du sol et l'utilisation des végétaux et enfin les notions de gestion et d'évolution. Au fil de ces questions, les différentes dimensions contextuelles, l'apport de références constituent des éléments de compréhension précieux.

9 À travers les différentes opérations, nous suivrons comme fil directeur le rapport au site; élément majeur de la conception des créations paysagistes de l'entre-deuxguerres, à première vue mis à mal dans les réalisations de l'après-guerre, et prenant depuis une vingtaine d'années la forme d'un leitmotiv incantatoire à travers la recherche de "la révélation » du site. En outre, nous aborderons la place accordée au paysagiste, l'évolution de son rôle dans l'interprétation de la question posée par le commanditaire et dans la définition des espaces ouverts par l'implantation des bâtiments ; enfin nous chercherons à cerner la posture du paysagiste dans le dessin des espaces à partir du matériau végétal, du travail du sol ou de la notion de temps. Matière première du projet paysagiste, la thématique du sol semble essentielle, plus encore que celle du végétal pour caractériser la pratique paysagiste.

Un métier partagé entre la pratique horticole et la conception de l'espace

10 À première vue, les réalisations de l'après-guerre furent, en France, peu soucieuses de prendre en compte la dimension du paysage autrement qu'à travers des pratiques compensatoires reposant sur le rôle "pacificateur " d'un végétal supposé racheter les excès d'une architecture jugée trop radicale. Cependant, la création d'une formation spécifique de paysagistes à l'École nationale d'horticulture de Versailles, au sortir du deuxième conflit mondial, laissait augurer d'une ambition plus complexe et d'une production plus diversifiée. Pendant la période étudiée, la formation versaillaise est la 
seule filière de formation diplômante et constitue, à ce titre et au moins comme point de départ, un reflet assez fidèle du milieu professionnel paysagiste. Ce nouvel enseignement délivre, selon les termes du décret «la double série de connaissances requises, celles de l'horticulture et celles de l'architecture ».

11 Ces deux pôles, qui ont pu être référés au scientifique et à l'artistique, caractérisent la formation et la culture des paysagistes ; une telle ambivalence au cœur de la discipline peut être vue tantôt comme complémentaire et constructive, tantôt comme contradictoire et ambiguë.

12 Le terme français "paysagiste", que les créateurs partagent ${ }^{5}$ avec entrepreneurs et pépiniéristes, distinct de l'«architecte-paysagiste " réservé aux concepteurs dans d'autres espaces géographiques et culturels, reflète pour la plupart d'entre eux, l'essence d'une pratique ancrée dans la réalité du sol et du climat, impossible à transmettre sans une forte implication dans la dimension physique des données locales. Ce titre ne permet pas de traduire les distinctions faites en anglais entre landscape architecture, landscape design et landscape planning et souligne la vocation de généraliste, construite sur l'idée de transformation volontaire de l'espace, revendiquée en France par les professionnels versaillais.

13 Situés à la charnière entre différents champs de pratiques, art, architecture, urbanisme et de disciplines, géographie, horticulture, sociologie..., les " paysagistes soucieux de n'être plus des "planteurs" mais des "concepteurs"" " sont souvent renvoyés à leurs propres contradictions, que ce soit à propos de leurs pratiques ou de leur processus de formation.

En se présentant comme spécialistes du végétal, les paysagistes placent en revanche une matière délicate et exigeante, puisque vivante, au cœur de leur pratique. [...] Mais, dès les années 1960, une évolution contradictoire se fait jour ; désireux d'asseoir un statut équivalent à celui des architectes, les paysagistes mettent de plus en plus l'accent sur la création et les techniques de projet, au risque de perdre leur spécificité7.

14 Cette dichotomie est particulièrement vive dans la période étudiée où il s'agit, pour les praticiens paysagistes, d'asseoir une identité en tant que concepteur, distincte de celle de l'entrepreneur, en faisant pencher la balance du côté de la création, tout en se démarquant des architectes et autres professionnels, en la ramenant du côté de la maîtrise du végétal et de son milieu.

L'héritage pluridisciplinaire des fondateurs de l'urbanisme

Dans l'entre-deux-guerres, une génération de professionnels8 de l'aménagement alliant précisément maîtrise du matériau végétal et culture de projet intervient au-delà des limites professionnelles et disciplinaires. À la fois ingénieurs, architectes, paysagistes et urbanistes, ils travaillent à l'ensemble des échelles d'intervention et mènent de front réflexion théorique et pratique du projet en associant approche esthétique et maitrise technique rigoureuse.

Dans leurs réalisations, la prise en compte des qualités du site est essentielle, le langage formel du projet s'appuyant sur le contexte particulier à chaque site. En témoignent notamment le parc Kellermann et le square René Le Gall conçus respectivement par Jacques Gréber à partir de 1937 et Jean-Charles Moreux en 1939 dans le $13^{\mathrm{e}}$ arrondissement de Paris, tous deux ancrés dans la topographie de la vallée de la Bièvre. La figure la plus emblématique de cette approche est celle de Jean-ClaudeNicolas Forestier $^{9}(1861-1930)$ qui expose sa vision du rôle structurant des espaces 
libres à l'échelle métropolitaine dans son ouvrage Grandes villes et systèmes de parcs ${ }^{10}$ en 1906. Bien que peu suivies d'effets dans la réalité des aménagements parisiens, ses idées sont reprises pendant la guerre, relayées ensuite par ses élèves, notamment Théodore Leveau (1896-1971) et Eugène Beaudouin (1898-1983) qui furent ses collaborateurs pour le plan d'urbanisme de La Havane entre 1926 et 1930 ; elles seront remises à jour dans les années $1980^{11}$.

Sous l'occupation allemande, au sein du service d'Urbanisme du département de la Seine, des paysagistes sous la direction d'Édouard Crevel et de Robert Joffet échappent au Service du travail obligatoire (STO) ; l'inventaire des espaces verts départementaux est dressé pour être complété selon les visions métropolitaines de J.-C.-N. Forestier. En l'absence de budget, les seules réalisations sont les terrains d'éducation physique (TEP), et les centres scolaires d'éducation physique (CSEP); ils constituent la base d'une ceinture de parcs, de squares et de terrains de jeux qui reprend le grand espace libre ${ }^{12}$ prévu dès 1912. Ces travaux forment le noyau du service des Parcs et Jardins dont R. Joffet sera conservateur après la guerre et à ce titre l'organisateur des Floralies de 1959, destinées à favoriser le goût du public pour l'art des jardins, quelque peu terni après la promotion des valeurs de retour à la terre portée par le régime de Vichy. En 1940, la création de l'Ordre des architectes interdit désormais aux paysagistes le port de leur titre favori d' " architecte-paysagiste ${ }^{13}$ ", confisquant ainsi une part de leur identité et renforçant leur mobilisation pour la revendication d'un titre et d'un diplôme spécifiques.

La Section du paysage et de l'art des jardins

18 La «Section du paysage et de l'art des jardins » est créée en 1945 à l'École nationale d'horticulture de Versailles sous tutelle du ministère de l'Agriculture afin de former des professionnels qui devront s'intégrer aux équipes de la Reconstruction, chargées du rétablissement de la France dévastée. Les futurs diplômés auront donc à se situer vis-àvis d'un double héritage; d'une part celui de J.-C.-N. Forestier et des pères de l'urbanisme moderne ${ }^{14}$, d'autre part celui du retour en force de la dimension horticole. Celle-ci s'était affirmée à la veille du conflit, notamment au cours de l'Exposition internationale des arts et des techniques appliqués à la vie moderne de 1937, en opposition à l'austérité de l'architecture moderne et aux excès de l'exposition ${ }^{15}$ de 1925.

Les premiers enseignants d'atelier, André Riousse (1895-1952) d'abord, puis Théodore Leveau, sont issus de cette culture de l'entre-deux-guerres et pratiquent un rapport modéré aux doctrines modernes. Ce dernier estime que les espaces verts associés aux quartiers organisés selon "l'ordre ouvert ${ }^{16}$ » - selon les préceptes de la Charte d'Athènes - nécessitent "un temps long, trente ans ", pour parvenir à un développement suffisant apte à constituer l'espace. En l'absence de moyens et de temps, il préconise de se tenir à un urbanisme d'îlots plus traditionnel ${ }^{17}$, ce qu'il formalisera dans sa contribution à la reconstruction des villes de Lille et Dunkerque.

André Riousse est architecte de formation, mais il a repris l'entreprise de jardins de son père et reçu un prix pour son projet de cour-jardin lors de l'exposition de 1925 ; il a participé à la conception de la Banlieue-jardin de la Butte Rouge à Châtenay-Malabry, réalisée à partir de 1931, à l'initiative d'Henri Sellier, créateur de l'OPHBMDS ${ }^{18}$. Un projet emblématique de l'art de l'implantation dans un site où se déclinent différentes échelles d'espaces collectifs associés à des dispositifs spatiaux accueillant une grande variété d'usages; intégration de plantations existantes ou association des nouveaux sujets à la composition urbaine, pièces d'eau, petits ouvrages maçonnés, treillages... 
conçus par le paysagiste et inspirés notamment par ses visites aux Siedlungen allemandes conçues par Ernst May.

21 Les problématiques paysagères sont proches des débats qui opposent alors les fonctionnalistes à leurs détracteurs, héritiers d'un urbanisme soucieux d'articulation historique et géographique dans la lignée de J.-C.-N. Forestier, dont J. Gréber, puis Gaston Bardet (1907-1989) et Robert Auzelle (1913-1983) reprennent le flambeau. Les préoccupations des paysagistes les rapprochent de cette filière de réflexion ; à l'inverse, on voit que leurs savoir-faire particuliers peuvent aussi relever d'autres champs professionnels, celui des architectes notamment.

22 Ainsi, autant que de ruptures, la période de l'après-guerre est tissée de figures de continuités. La pratique des paysagistes les ramène au sol, au terrain, aux qualités locales des sites en même temps qu'elle les ouvre à des logiques d'aménagement d'échelles plus larges. L'héritage des jardins versaillais les associe aux représentations nationalistes, tandis que l'uniformité des espaces verts modernes renvoie à l'abstraction artistique. Les situations offertes, les programmes proposés et les questions soulevées sollicitent abondamment la dimension du paysage, interprétée alors en termes de cadre de vie; libération du sol, ouverture de l'espace, développement des activités de loisirs, régionalisme, abstraction du territoire... Dans la période qui nous occupe, le volet paysage des projets d'aménagement en milieu urbain passe de l'ouverture de l'espace vert moderne à l'étendue du territoire.

Une génération confrontée à de nouveaux enjeux, à une nouvelle échelle d'intervention L'examen des réalisations paysagistes de l'après-guerre permet d'identifier trois étapes correspondant chacune à une décennie environ, de 1945 à 1975. Dans un premier temps, 1945-1955, pragmatisme et invention permettent d'adapter à l'échelle du grand ensemble les pratiques d'aménagement de squares et de parcs acquises précédemment.

Puis, de 1955 à 1965, les nouveaux diplômés accèdent peu à peu à la commande, mais, en dépit des objectifs de création de la Section du paysage, restent le plus souvent cantonnés à des tâches de verdissement des grands ensembles. Parvenue à maturation, la nouvelle génération porte sur l'enseignement comme sur les pratiques professionnelles françaises un regard critique enrichi du contact des cultures d'Europe du Nord.

Enfin, à la fin des années 1960, dans la mouvance des réflexions pluridisciplinaires qui inspirent alors le milieu architectural français, un revirement se produit dans la culture de référence des paysagistes. L'enseignement versaillais est assuré, de plus en plus, par des personnalités issues des milieux artistiques - peinture, décoration, architecture, mobilisées par des problématiques de composition. De médiateur entre l'homme et la nature, le paysagiste devient un concepteur imposant son écriture au milieu existant. La dimension technique, horticole et botanique, jusque-là dominante, passe progressivement au second plan, avant de se voir considérée plus tard, à la fin des années 1980, comme élément fondateur de la pratique paysagiste.

Pragmatisme et invention

Dans l'euphorie de la Reconstruction, la première série d'opérations, de 1945 à 1955, est portée par la foi dans la modernité et le progrès. Deux d'entre elles appartiennent au "Secteur industrialisé ", programme d'innovations lancé par le ministère de la Reconstruction et de l'Urbanisme en 1951 dont les objectifs sont centrés sur la « cellule » du logement et l'industrialisation du bâtiment. L' « espace vert », supposé abondant, hygiénique et fonctionnel, assure la promotion de ces opérations à l'aide de 
slogans prometteurs comme ; " Habiter dans un parc ", mais reste le parent pauvre des préoccupations des architectes et des maîtres d'ouvrages.

À Beaulieu le Rond-Point, au sud de Saint-Étienne, 1220 logements s'inscrivent avec virtuosité dans un terrain en forte pente. À la cité de l'Aubépin, à Chalon-sur-Saône, les bâtiments s'organisent autour d'un grand espace central, sur le site d'une ancienne tuilerie où les excavations argileuses sont transformées en pièces d'eau poissonneuses. Mais à Bron-Parilly en périphérie lyonnaise, à la croisée de futures autoroutes, rien n'a été prévu entre bâtiments et voieries séparés par de grands dénivelés.

Dans chaque cas, le recours au paysagiste se fait après que les bâtiments sont réalisés. Le plan d'ensemble à Beaulieu le Rond-Point suit les préceptes de l'art urbain prôné par R. Auzelle, de même que l'espace ménagé au centre de l'Aubépin rappelle celui qu'E. Beaudouin conçut pour le projet lauréat du concours de Strasbourg en 1951. Si ces deux opérations relèvent d'une organisation guidée par l'association de préceptes modernes et d'un souci de composition des espaces, Bron-Parilly en revanche souscrit sans compromis aux règles de «l'ordre ouvert » inspiré de la Charte d'Athènes.

Ces situations reflètent la diversité et les contradictions de l'époque entre idéaux modernes et recours à des héritages plus anciens, faute de savoir-faire mieux adapté, la doctrine de la Charte d'Athènes s'avérant singulièrement muette en la matière ${ }^{19}$. Ce décalage entre modernité et tradition apparaît encore accentué dans le milieu paysagiste peu impliqué, nous l'avons dit, dans les réflexions modernes ${ }^{20}$.

Dans la première décennie qui suit la Libération, la culture de l'entre-deux-guerres perdure tout d'abord, notamment à travers l'intérêt porté aux qualités du site comme base de conception. Cette continuité peut traduire l'intérêt porté par l'humaniste Raoul Dautry, qui est alors le premier à diriger le ministère de la Reconstruction et de l'Urbanisme, à la dimension du paysage, voire même l'amorce d'une politique du paysage, dont la création de la Section du paysage serait l'expression, au-delà de la simple professionnalisation des paysagistes. Une ouverture sans doute vite compromise, selon les mots de Bernard Barraqué, par son successeur «Eugène Claudius-Petit ${ }^{21}$ (qui) semble avoir, après 48 , globalement tranché en faveur de Le Corbusier et de ses épigones ${ }^{22}$ ». Cette hypothèse reste à confirmer.

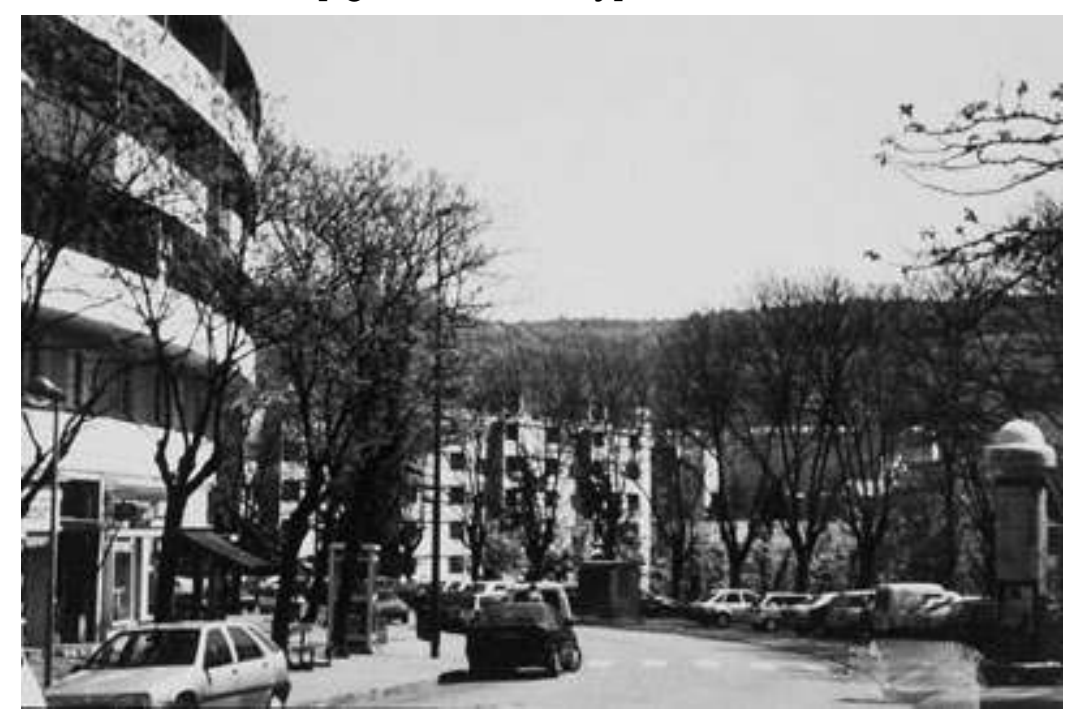


«Exemple nécessitant l'intervention d'un paysagiste ", mentionne la plaquette de la Section du paysage en légende d'un croquis de la maquette de Beaulieu le Rond-Point. Le paysagiste Jean Marc, formé à l'École nationale d'horticulture en 1922, accompagne l'ensemble d'habitations d'une déclinaison de haies, contre-haies, arbustes et arbres colorés et rythmés qui se glissent aussi dans les lointains du paysage local de bouchures ${ }^{23}$. Ce méridional d'origine alsacienne a lu Le Corbusier et visité l'exposition de 1925 ; c'est avec pragmatisme et patience qu'il adapte à l'échelle du grand ensemble les principes du square urbain acquises à l'École nationale d'horticulture. Les aménagements sont réalisés en régie ${ }^{24}$, sans dessin préalable, et suivent les tracés dessinés par l'usage. Ils prennent le relais des prairies fauchées et pâturées au pied des bâtiments, longtemps après que les premiers habitants se sont installés. Leur foisonnement et leur structure constituent une véritable écriture déclinée par le paysagiste dans tout le département. Ils ont d'ailleurs inspiré plus tard la réhabilitation de la ZUP de Montreynaud (1967), autre grand ensemble stéphanois, plus tristement caractéristique des réalisations de la période des Trente Glorieuses. L'Atelier d'urbanisme et d'architecture (AUA), avec le paysagiste Alexandre Chemetoff proposera là, à partir de 1978, une restructuration exemplaire, qui renoue avec les qualités des cités-jardins des années 1930 comme celle de la Butte-Rouge.

32 À la cité de l'Aubépin, le paysagiste utilise des techniques de plantations forestières, avec de jeunes plants et des semis de trèfle blanc, pour cerner de masses denses les clairières de jeu qu'il implante au sein du grand espace central. Henri Pasquier fut formé à l'École du Breuil dans les années 1920, il mena ensuite une thèse à l'Institut d'urbanisme de Paris. Pilier du service de R. Joffet, il obtint le titre de paysagiste DPLMA en 1946 et participa ensuite à la création de l'École supérieure d'art des jardins $(\mathrm{ESAJ})^{25}$. Forte personnalité, auteur et rédacteur de nombreuses publications, notamment dans la revue Urbanisme, très impliqué dans la promotion de la profession, il défendit l'intervention du paysagiste dans l'espace public, les grands ensembles ou les abords d'autoroutes.

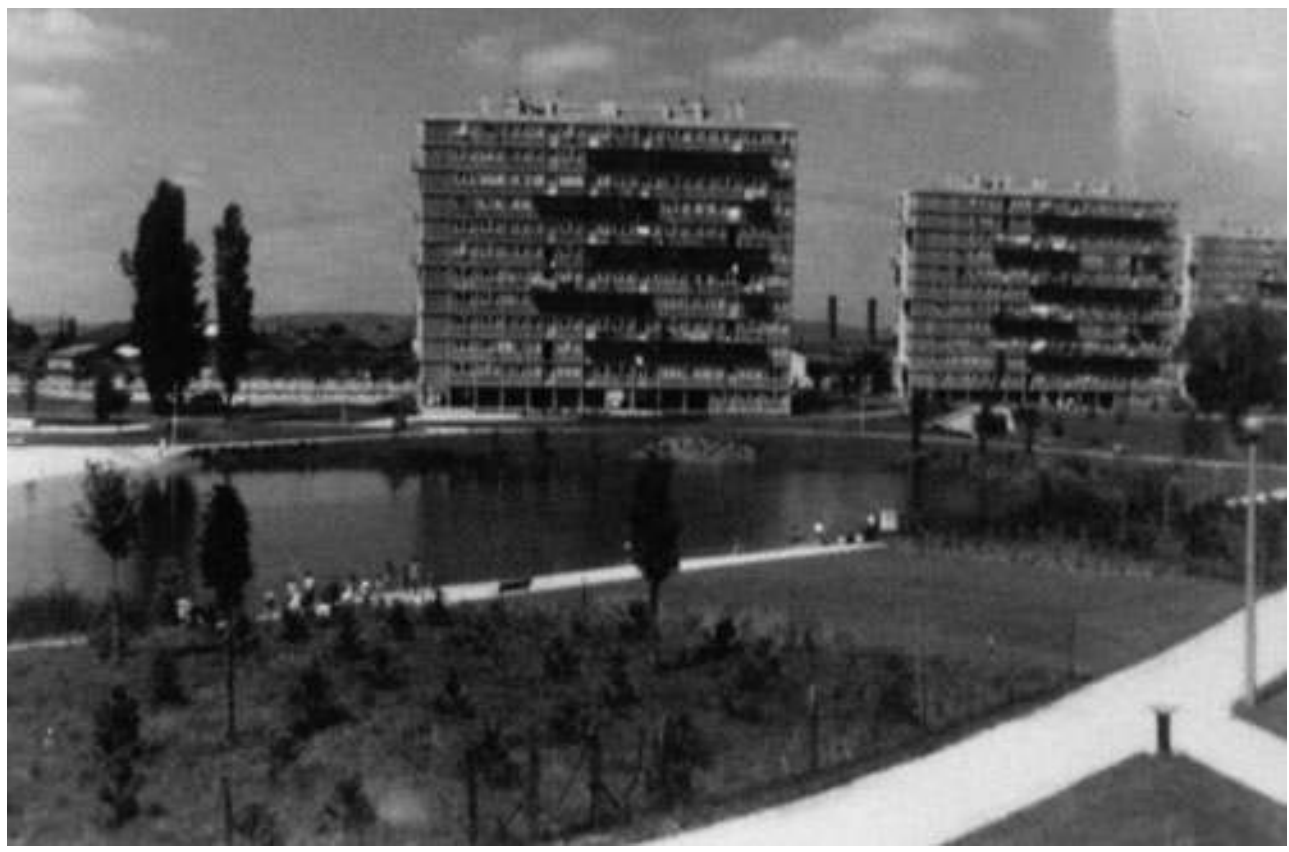


Cité de l'Aubépin. Paysagiste : Henri Pasquier @ André Pelletier.

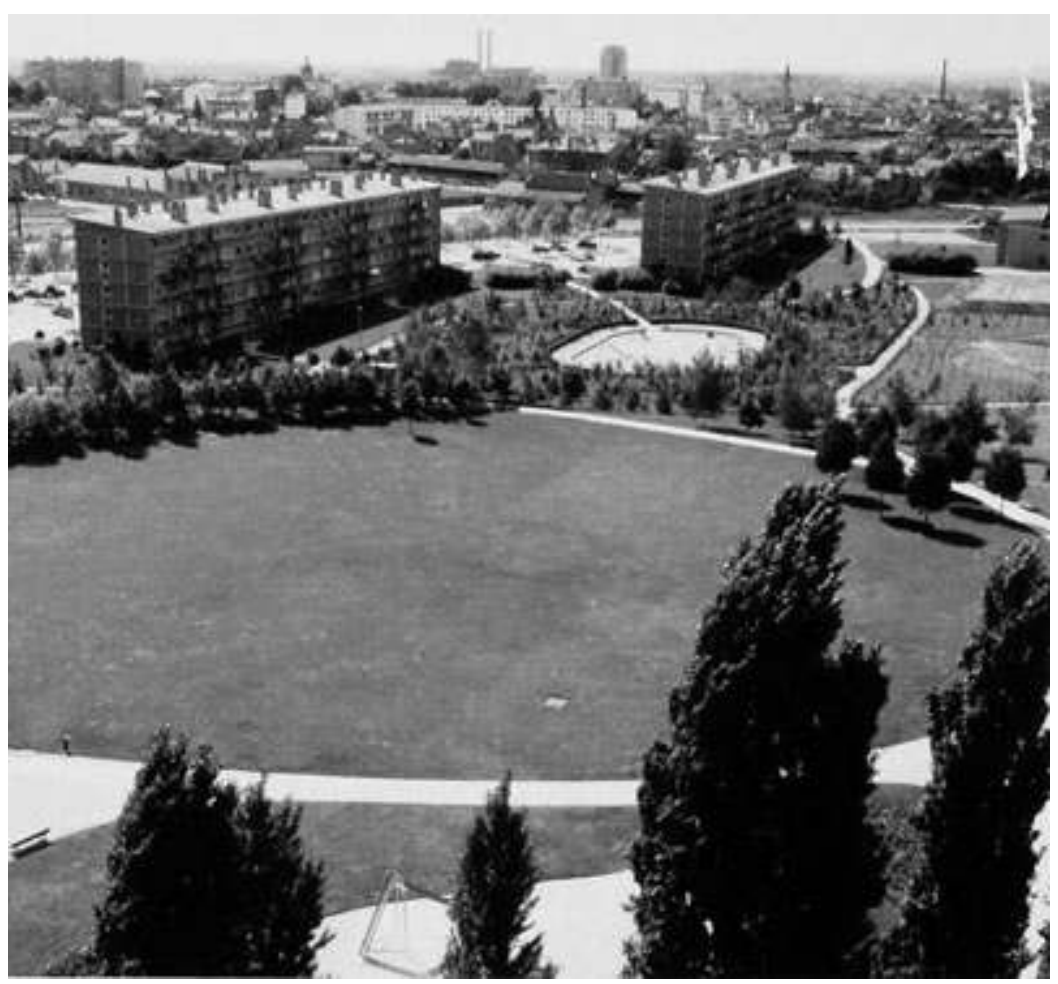

Cité de l'Aubépin. Paysagiste : Henri Pasquier @ André Pelletier.

Nous l'avons dit, aucun de ces praticiens ne fut associé au travail de plan de masse ni à l'interprétation de la commande. Mal définie, souvent réduite à une injonction du type «arrangez-nous ça », celle-ci traduit l'inexpérience que les maîtres d'ouvrage partagent avec les concepteurs pour cette nouvelle échelle d'intervention. À l'Aubépin, H. Pasquier fut à l'origine de la création du premier service d'Espaces verts au sein d'un office d'HLM, modèle alors pour la France, qui inspira la création de celui de SaintÉtienne par J.Marc. On doit aux organismes d'HLM de nombreux projets d'aménagement extérieurs de grands ensembles, achevés grâce aux premiers loyers pourtant destinés à alimenter les budgets d'entretien! Mais bien souvent les aménagements extérieurs n'ont simplement pas été réalisés. 


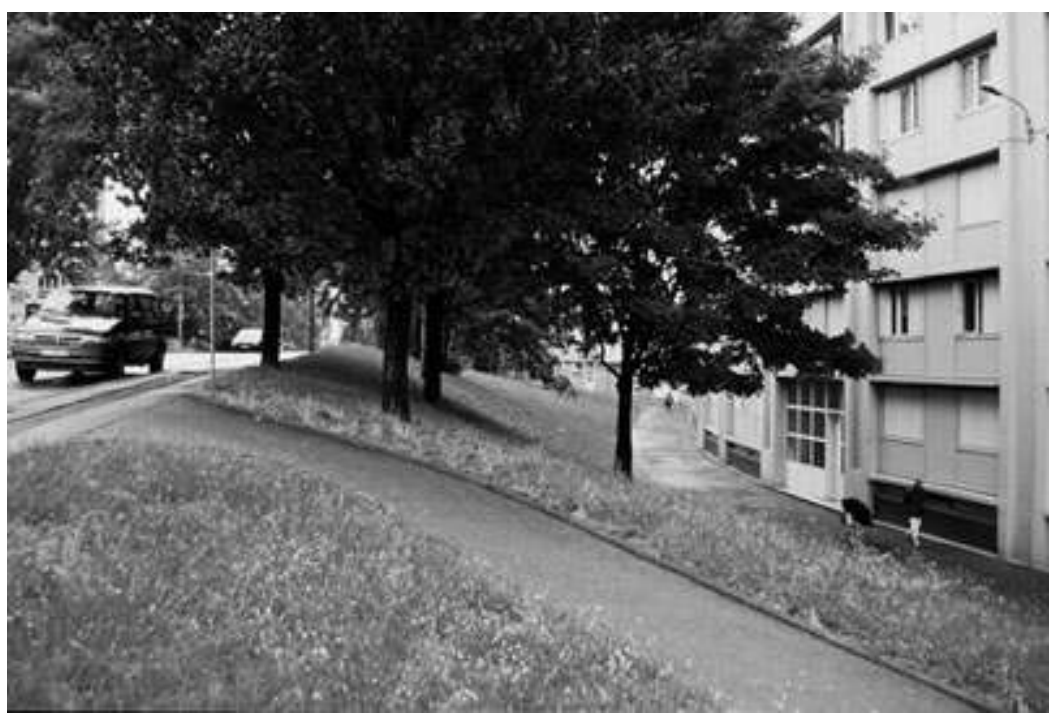

Bron Parilly. Paysagistes : Michel et Ingrid Bourne @ B. Blanchon. évoluer la commande, de projet en projet. Paradoxalement, le désintérêt des architectes pour les aménagements extérieurs a permis à la pratique paysagiste de s'aiguiser à l'abri des concurrences et des remises en cause. En acceptant le défi qui leur fut soumis, Michel et Ingrid Bourne, jeunes diplômés de la Section du paysage, engagèrent un long processus de démonstration pour convaincre de la justesse de leur démarche et imposer le recours au paysagiste en amont du projet.

-Parilly, le site se résume aux bâtiments; pour relier bâtis et routes séparés parfois par cinq mètres de dénivelés et en l'absence de relevé de géomètre, M. et I. Bourne utilisent des engins de travaux publics maniés en régie, complétés par des semis agricoles; ils abandonnent les haies qui ne leur semblent pas adaptées à l'échelle des bâtiments et cherchent à réaliser les masses vertes suggérées dans les œuvres de Le Corbusier. Issu d'une grande famille de pépiniériste, M. Bourne sollicite la pépinière paternelle afin d'adapter les essences choisies aux sols stérilisés par le compactage des engins. Là, comme à l'Aubépin, la mécanisation de pratiques jusqu'ici restées artisanales, le recours aux techniques modernes permettent de s'adapter à cette nouvelle échelle de travail.

Le paysagiste comme médiateur entre l'homme et la nature

La deuxième série d'opérations, 1955-1965, prend place alors que les doctrines modernistes commencent à être, sur le plan théorique et idéologique, battues en brèche et remises en cause; lors du dixième CIAM à Dubrovnik en 1956, Team 10 critique la Charte d'Athènes comme anachronique et simpliste. Plus concrètement, cette deuxième série d'opérations s'inscrit dans le contexte français à la fois du développement et de la critique naissante des grands ensembles. Durant l'hiver 1954, l'appel de l'abbé Pierre a donné l'impulsion nécessaire à l'essor de la politique du logement portée par le Plan Courant ${ }^{26}$ (1953). Les objectifs quantitatifs atteints ${ }^{27}$ en matière de réalisation de logements, l'enthousiasme s'accompagne d'une inquiétude grandissante sur la qualité de la vie dans ces ensembles de logements. Pierre Sudreau, qui accède au ministère de la Construction en 1958, a créé auparavant une commission 
sur « Les problèmes de la vie dans les grands ensembles d'habitation ${ }^{28}$ ", dont est issue la grille d'équipements connue sous le nom de grille Dupont.

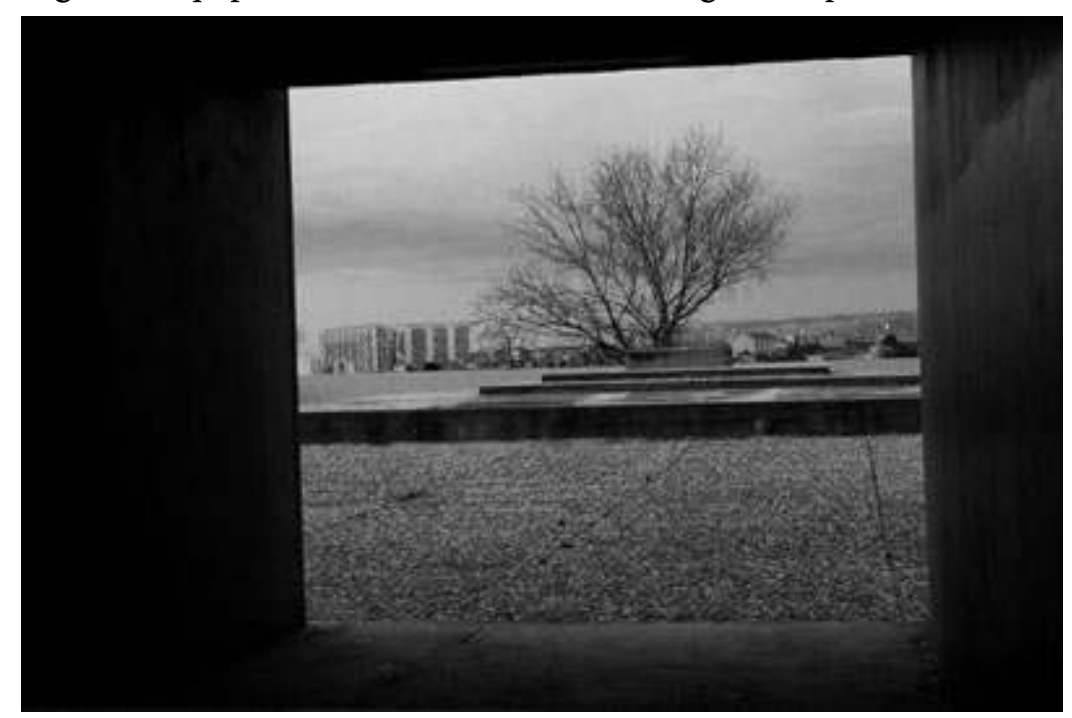

La Maurelette. Paysagiste : Jacques Sgard et coloriste : Bernard Lassus @ B. Blanchon.

37 La réalisation de l'unité de voisinage de la Maurelette, dans les collines du nord de Marseille, mise à l'étude dès 1959 et voulue d'emblée exemplaire, reflète particulièrement bien ce contexte. À Grenoble, au pied des montagnes, l'opération du Village Olympique s'inscrit entre dalle et sol naturel, dans l'épaisseur d'une ancienne plaine agricole; en périphérie lyonnaise, la deuxième tranche des Minguettes se démarque de l'urbanisation dense du plateau, en cherchant à intégrer les tours dans une pente orientée au sud-ouest. Les paysagistes associés à ces opérations sont tous diplômés de la nouvelle formation versaillaise. Pour deux au moins de ces opérations, ils sont présents dès le début de l'opération et participent à l'implantation des bâtiments ; une reconnaissance vis-à-vis des commanditaires et des architectes obtenue à force de travail et d'exigence sur le terrain.

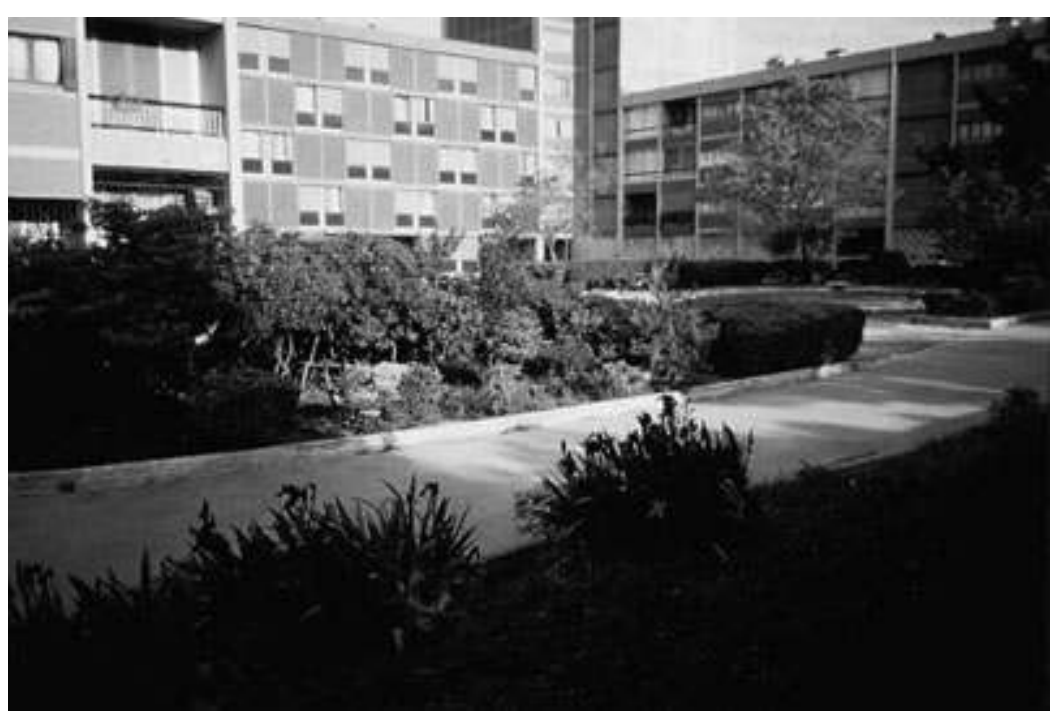


À la Maurelette, Jacques Sgard issu de la deuxième promotion (1947) de la Section du paysage est appelé grâce à son approche remarquée aux 4000 de La Courneuve - bien que peu suivie d'effet -, où il a cherché à structurer le quartier à travers différentes échelles d'espaces plantés. Il est associé dès le début aux réunions préliminaires organisées par le Comité interprofessionnel du logement (CIL) auxquelles participent outre les architectes, médecins, psychologues, assistantes sociales et même un coloriste en la personne de Bernard Lassus, élève du peintre Fernand Léger. Le programme est bientôt réduit de 1500 à 750 logements, les deux unités d'habitation initialement prévues sont oubliées, pour rechercher la constitution d'espaces de voisinage, d'échelles de convivialité. J. Sgard et B. Lassus sillonnent la campagne pour s'imprégner des caractéristiques du paysage local. Les architectes Eugène Chirié et ses fils Pierre et Jacques, militants régionalistes, étudient les caractéristiques locales de l'architecture en réponse aux contraintes du mistral et de l'ensoleillement ; ils s'inspirent notamment des cours d'Aix-en-Provence et d'Aubagne. Adeptes de Le Corbusier à Ronchamp ou à La Tourette, ils critiquent l'Unité d'habitation et cherchent à faire des « redondances de plan-masse ». Ils s'intéressent aux recherches de Georges Candilis, représentant du Team 10, pour la conception du plan-masse sous forme de trame, afin de créer des espaces d'échelles intermédiaires. Il est intéressant de noter que Michel Ecochard, responsable du Service de l'urbanisme du Protectorat français au Maroc, qui permet à l'équipe de G.Candilis d'expérimenter ses premières réalisation dans le cadre de l'ATBAT-Afrique, appelle à travailler avec lui, dès leur sortie de l'école, les premières promotions de la Section du paysage ${ }^{29}$.

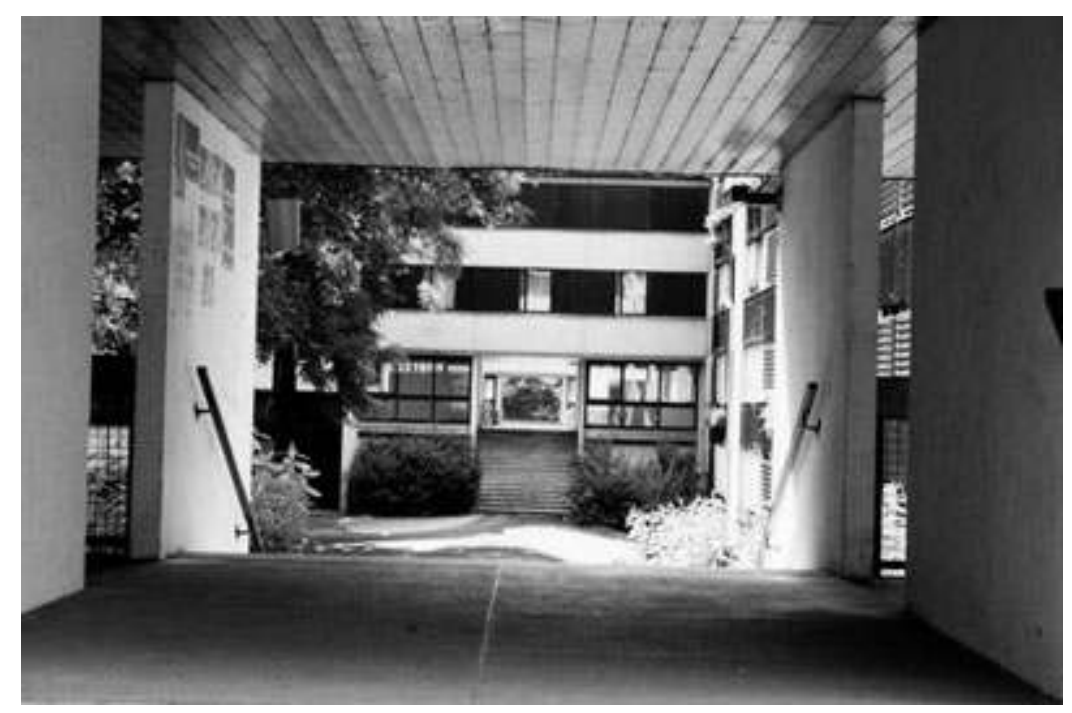

Le Village olympique de Grenoble. Paysagiste : Jean-Claude Saint Maurice $\odot$ B. Blanchon.

Réalisé à partir de 1962, le projet de l'Unité de voisinage de La Maurelette intègre les caractères locaux du paysage ; mails, traboules et passages tissent un réseau de vues et de placettes minérales autour des éléments existants; une allée de platanes, une bastide et sa tèse ${ }^{30}$. Les feuillus à larges feuilles sont privilégiés pour ménager ombre et fraîcheur ; tout comme l'orientation sud des façades principales pour éviter le mistral à l'est et l'excès de soleil à l'ouest. Il n'est pas prévu de déblais afin de ménager l'hydrographie du site ${ }^{31}$, les bâtiments, parkings compris, sont posés directement sur le 
sol existant. Initialement prévue en bordure de rue, pour faire le lien avec le village Saint-Joseph, l'implantation du centre commercial se plie aux prescriptions de l'architecte-conseil parisien et intègre le cœur de l'opération - où il périclite aujourd'hui. Pour les façades, B. Lassus développe un travail fin de pâtes de verre colorées, destiné à lier les bâtiments au site - à «cielliser» les tours, et éviter la réverbération.

La collaboration de B. Lassus et de J. Sgard se poursuivit sur le grand ensemble de Quétigny-les-Dijons, projet également porté par une maîtrise d'ouvrage éclairée, ainsi que dans l'enseignement de la Section du paysage et de l'art des jardins de Versailles. La Maurelette est une opération expérimentale voulue exemplaire, mais qui malgré son succès, ne fut pas renouvelée; pourtant, plus tard de nombreuses cités dans les quartiers nord seront réhabilitées, y compris avec le concours de paysagistes, alors que La Maurelette n'a nécessité qu'un entretien légitime.

41 Le souci de la qualité pédologique du substrat, comme condition de la maîtrise du végétal, tout comme l'attention portée au nivellement comme base de la constitution des espaces de voisinage est constant dans ces opérations. Au Village Olympique de Grenoble, Jean-Claude Saint Maurice fait déplacer la terre végétale afin d'éviter son tassement ; son projet converse doucement avec les bâtiments, modelant un sol tantôt naturel, tantôt sur dalle tout en préservant l'unité de l'aménagement dont la pérennité s'ancre dans un travail minutieux du substrat. La strate du sol s'affirme comme le lieu privilégié du projet paysagiste, sa fondation même.

Aux Minguettes, forts de leurs expériences passées dans la plupart des grands ensembles de la région, M. et I. Bourne revendiquent l'implication du paysagiste en amont du plan de masse. Ils défendent la préservation d'un grand «vide » central comme celui qu'H. Pasquier appréciait à l'Aubépin -, figure souvent retrouvée lorsque le paysagiste est impliqué dans l'élaboration du plan d'ensemble. Cette ouverture, garante d'une liberté d'usages est essentielle à préserver ; elle doit être aujourd'hui défendue face à la propension des services de ville à remplir les espaces vacants sans en comprendre le sens. La disposition des tours ménage des vues sur le coteau opposé, la strate arborée s'inscrit dans une échelle intermédiaire entre l'homme et les bâtiments. Comme à l'Aubépin et dans de nombreux projets, la structure des modes de plantations accompagne celle des bâtiments; mails et plantations d'alignement ceinturent les opérations - associées à des charmilles aux Minguettes - et absorbent les stationnements en périphérie, des haies structurent les abords de bâtiments accompagnées de lisses béton aux Minguettes tandis que des formes plus libres se dessinent lorsque l'espace s'éloigne de l'influence des bâtiments. 


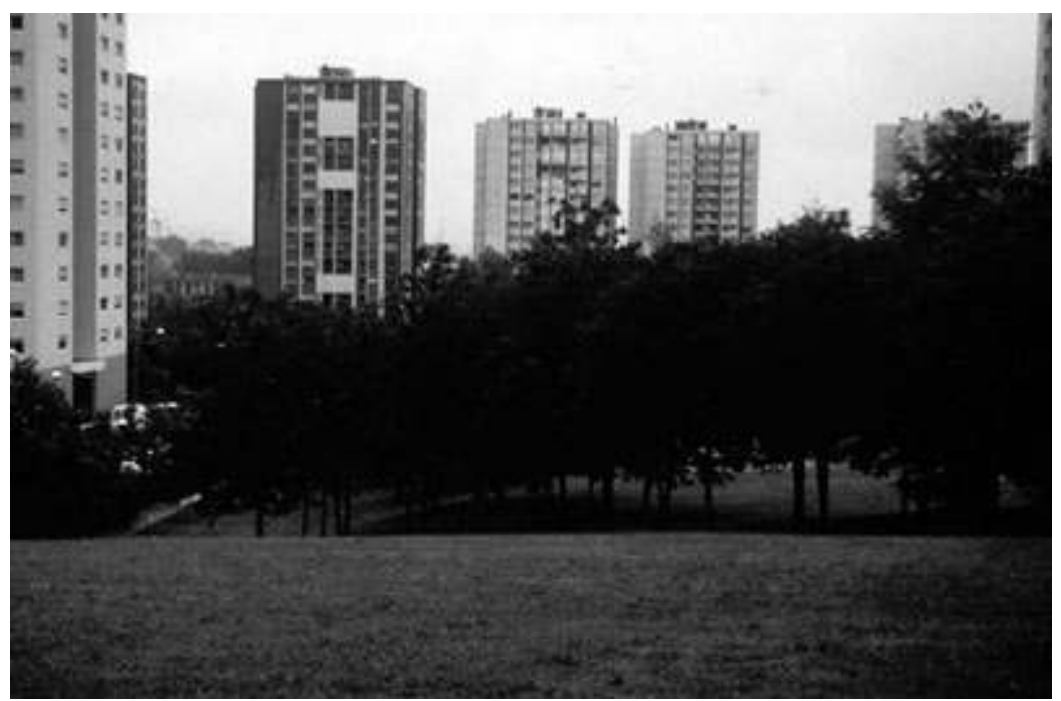

Les Minguettes. Paysagistes : Michel et Ingrid Bourne ㄷ B. Blanchon. présence de personnalités telles qu'A. Riousse et T. Leveau, les élèves paysagistes de la Section du paysage recherchent des compléments de formation à l'Institut d'urbanisme de l'Université de Paris où enseignent R. Auzelle et Jean Royer. C'est dans leurs contacts avec les pays d'Europe du Nord qu'ils puisent leurs modèles de réflexion et de pratique.

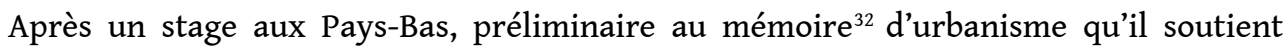
en 1958, J.Sgard introduit en France le paysage d'aménagement ${ }^{33}$ dont il reste aujourd'hui le principal propagateur. L'agence qu'il fonde avec deux collègues est le creuset d'une réflexion qui s'inscrit dans cette perspective pluridisciplinaire, écologique et environnementale. Tandis que d'autres jeunes diplômés séjournent au Danemark $^{34}$, M. et I. Bourne mettent à profit dans leurs projets l'expérience des réalisations allemandes apportée par I. Bourne ${ }^{35}$; les autoroutes du $\mathrm{III}^{\mathrm{e}}$ Reich d'Alwin Seifert comme les opérations de logements ou le jardin botanique de Hambourg réalisés dans les années 1930. À l'instar de leur maître Karl Plömin, ils réalisent des relevés de milieux locaux afin de comprendre et reproduire les associations végétales correspondant aux terrains qu'ils abordent.

À Versailles, dès 1963, la jeune génération investit l'enseignement à son tour et obtient en 1967 la création d'un cours d'écologie avec Jacques Montégut - titulaire de la Chaire de botanique à l'école nationale d'horticulture et qui fût à l'origine de l'intérêt d'un Gilles Clément pour le végétal; J. Sgard apporte son intérêt pour les démarches dites de Grand paysage et introduit le plasticien B. Lassus dans l'enseignement de la section.

Ces jeunes professionnels ont participé à la réflexion sur l'évolution des grands ensembles ${ }^{36}$ ainsi qu'aux différents organismes publics ${ }^{37}$ d'aménagement du territoire. J.-C. Saint Maurice notamment, qui participa aux travaux de la Commission de la vie dans les grands ensembles et réalisa de nombreux projets pour la Société civile immobilière de la Caisse des dépôts et consignations (SCIC) et la Société centrale d'équipement du territoire (SCET), défend l'implantation de jeux de ballon informels accessibles aux jeunes, pour tout groupe de 300 habitants. Il développa ses idées sur le rôle social de l'espace vert dans la recherche «Les territoires de vie quotidienne de $l^{\prime}$ habitat ${ }^{38}$ ", qu'il réalisa pour le plan urbain. Ils furent nombreux à intégrer les équipes des Organisations d'études et d'aménagement des aires métropolitaines (OREAM);

Strates, $13 \mid 2007$ 
J. Sgard, notamment, participe aux OREAM de Lorraine et de Marseille ainsi qu'à l'organisation d'étude et d'aménagement de la région Alsace (OEDA).

Tous défendent une conception au service des usagers et des maîtres d'ouvrage, privilégiant avant tout une réflexion fondée sur l'usage des aménagements proposés. Ils fondent leur approche sur leurs capacités d'observation et de lecture du terrain. Ils associent la connaissance profonde des processus qui conduisent à la formation d'un paysage à une réflexion prospective, articulant ainsi la dimension de l'espace et celle de la durée. Les bases de conception des projets qui s'élaborent alors reposent sur une maîtrise technique et sur une compétence revendiquée dans le domaine du végétal une compétence considérée de plus en plus comme le laboratoire du travail sur l'échelle du territoire.

Pourtant, l'influence des cultures paysagistes du Nord de l'Europe deviendra ensuite minoritaire dans la formation versaillaise et la pratique professionnelle, laissant place à une culture plus architecturale et urbaine.

La maîtrise de la composition, l'autonomie du projet de paysage

Dans la dernière décennie, 1965-1975, la fin de la pénurie de logements s'accompagne d'une sévère critique, de la part de la société, du gigantisme d'une urbanisation trop uniformisée. La « circulaire Guichard » met fin aux grands ensembles en 1973 ; la même année, le premier choc pétrolier annonce la fin de la période d'expansion économique. Dans l'effervescence intellectuelle qui entoure Mai 1968, la pensée architecturale se ressource dans l'histoire et dans l'intérêt pour la complexité de la ville. Répondant à la remise en cause de la profession, de nouvelles structures associent différentes disciplines dans la conception du bâtiment; c'est le cas notamment de l'AUA, fondé en 1960 par l'urbaniste et sociologue Jacques Allégret.

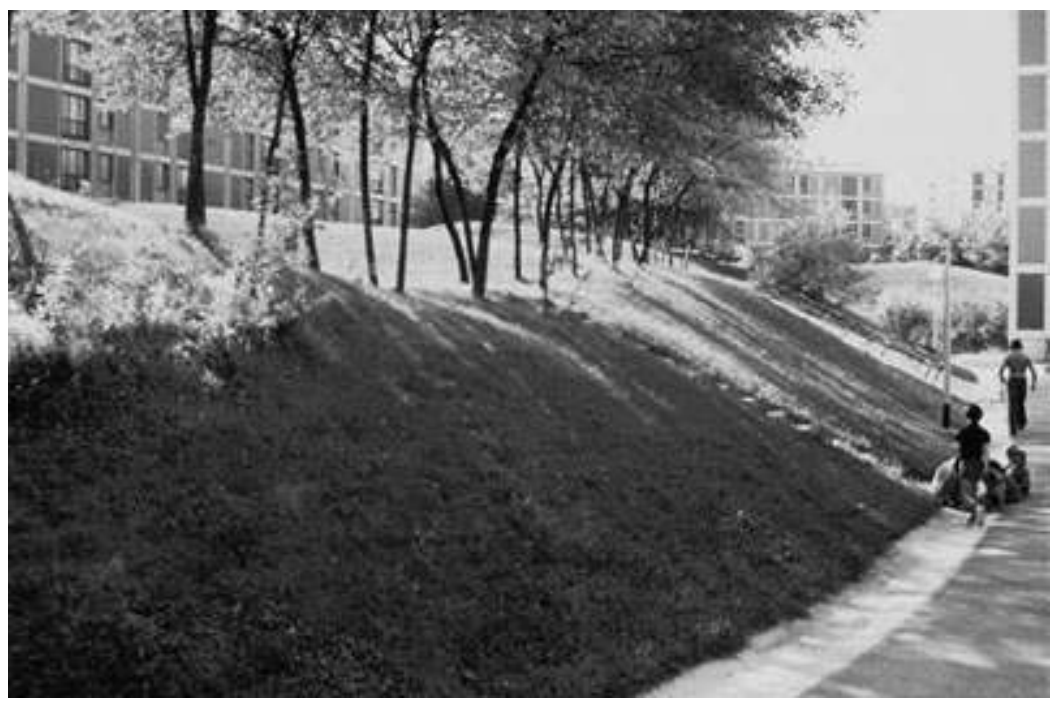


Reims. Zup des Châtillons, avant le réaménagement de 1999. Paysagiste : Jacques Simon ${ }^{\circ}$ A. Chemetoff.

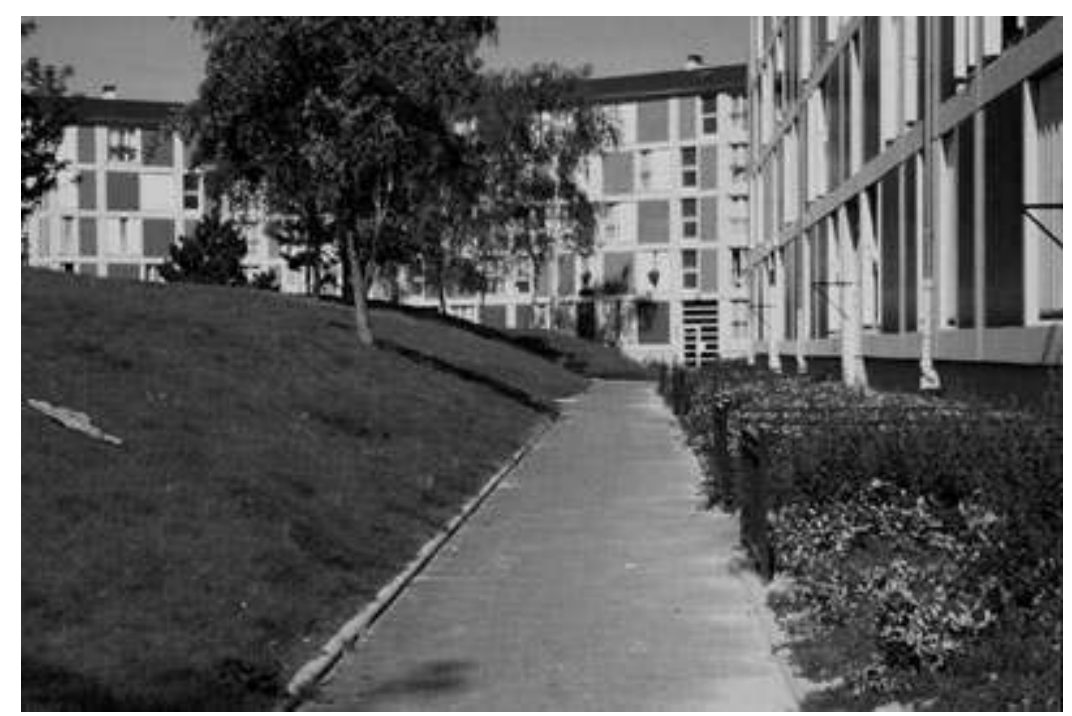

Reims. Zup des Châtillons, avant le réaménagement @ B. Blanchon.

Au parc Saint John Perse, à partir de 1967, à la périphérie de Reims, la commande se résume à dégager le terrain destiné à une future construction; non loin, à la ZUP des Châtillons, là encore, les bâtiments tiennent lieu de site. Leur forme d'écrous constitue des «chambres" à aménager et à relier entre elles. À la Villeneuve de Grenoble, la jeune équipe de l'AUA est appelée pour renouveler la réflexion sur l'extension sud de la ville. Proposé comme un modèle urbain novateur, le bâtiment développe une rue haute en balcon sur le parc qu'il enserre. Dans toutes ces opérations, les déblais des constructions sont la base d'une nouvelle topographie, d'un nouveau sol conçu par les paysagistes.

Jacques Simon a suivi l'enseignement de la Section du paysage de 1957 à 1959 et mené ensuite en Suède, puis au Canada, des études à la fois horticoles et artistiques; l'exercice du métier de bûcheron l'a convaincu que l'on ne peut se fier au végétal pour structurer l'espace. Collaborateur régulier de l'AUA, il y rencontre Michel Corajoud, alors élève aux "Arts déco ", qu'il forme et convertit au paysage et qui prendra plus tard sa place au sein de l'AUA; leur tandem jouera un rôle essentiel dans la diffusion d'une culture française du projet de paysage. 


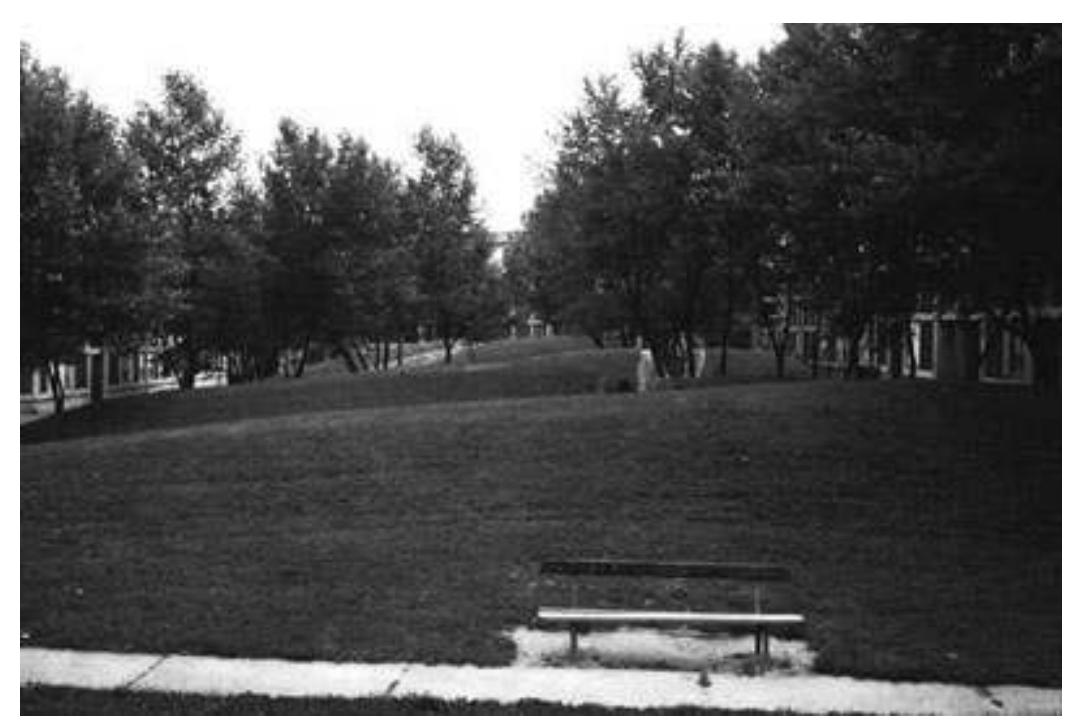

Reims. Zup des Châtillons, avant le réaménagement de 1999. Paysagiste : Jacques Simon $\odot$ A. Chemetoff.

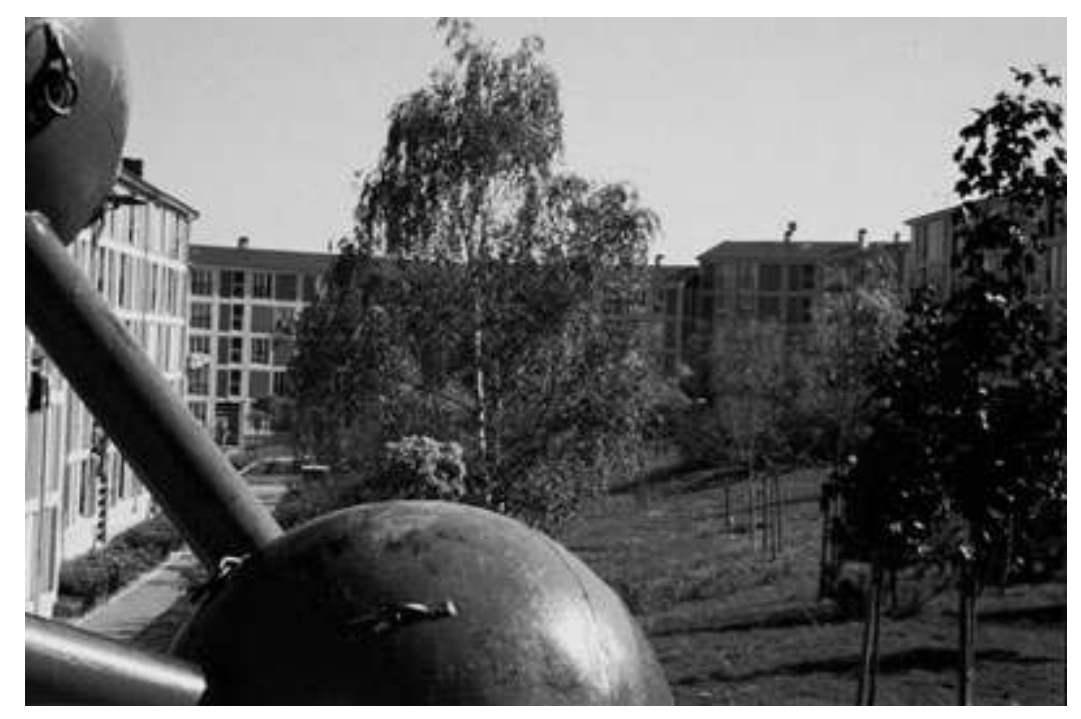

Reims. Zup des Châtillons, avant le réaménagement @ B. Blanchon.

51 Aux Châtillons, J. Simon revendique l'autonomie du projet de paysage dont l'impact doit égaler en force celui de bâtiments peu soucieux d'intégration. Pragmatique, il relativise ainsi l'impact d'un recours tardif au paysagiste. À partir d'éléments simples, il réinvente un paysage autonome, ludique et sensuel où l'homme donne la mesure; les intérieurs d'îlots sont reliés par un mail de paulownias et crépitent d'oiseaux, d'arbres en fleurs et d'activité humaine; de fortes buttes, chevauchées par des peupliers-neige et cernées de caniveaux accueillent des jeux d'enfants conçus par le paysagiste. Son approche s'ancre dans un travail de terrassements destiné à garantir la structure de l'espace dans la durée. Mais l'émotion provoquée par cet aménagement généreux n'est plus au rendez-vous aujourd'hui, du fait d'une réhabilitation récente malheureuse.

52 Travaillant sans dossiers à même le terrain, J. Simon se confronte au site; au parc Saint John Perse, il installe une grande chambre verte vouée aux rassemblements humains. Il entaille l'ourlet planté qui l'entoure dans la direction d'une chapelle 
voisine, retissant ainsi les liens avec le contexte. La figure de J. Simon, insaisissable, atypique, photographe volant, éditeur, en prise avec les éléments de la planète et l'ensemble des acteurs de l'aménagement fait l'articulation entre deux générations.

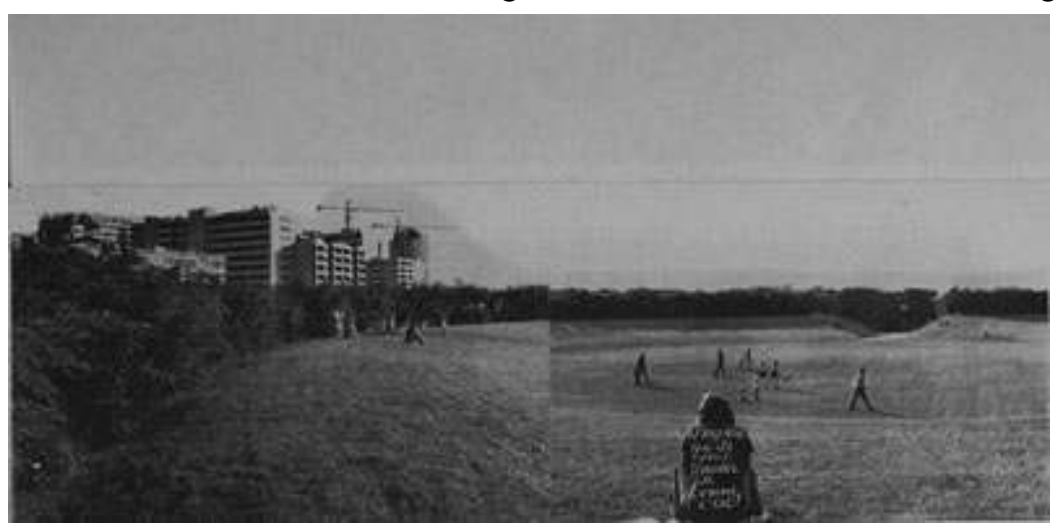

Parc St John Perse, Reims. Paysagiste : Jacques Simon @ Collage photos Jacques Simon.

À la Villeneuve de Grenoble, l'équipe $\mathrm{CCH}^{39}$, paysagistes urbains au sein de l'AUA, installe une superposition de trames d'échelles variées où la nature est passée au filtre de la géométrie. Ignorant les montagnes voisines, un nouveau paysage de buttes parcourues de lignes d'arbres serpentant comme des rivières, recouvre la plaine alluviale. Le projet englobe bâtiments et parc dans une vision commune. Le parc est conçu comme un équipement en plein air, comme le lieu de pratiques nouvelles. M. Corajoud milite pour attribuer aux paysagistes des prérogatives équivalentes à celle de l'architecte. Il critique chez ses prédécesseurs ce qu'il considère comme des pratiques compensatoires, destinées à racheter l'excès de béton dans une vision antiurbaine qu'il combat. Les références ${ }^{40}$ utilisées basculent vers les traditions latines, les terrasses cultivées et les jardins de Le Nôtre.

Malgré le développement des préoccupations environnementales et la participation de nombreux paysagistes aux équipes des OREAM et des Villes Nouvelles, la Section du paysage doit fermer en 1974, alors que le Centre d'études et de recherches en paysage (CNERP) a été créé en 1972 à la demande de Robert Poujade, premier ministre de l'Environnement, afin de former des "paysagistes d'aménagement». Appelé par Jacques Simon et Pierre Dauvergne pour enseigner à Versailles, M. Corajoud a formé à partir de 1970, dans un contexte instable, une génération de professionnels reconnus dans les années 1980. Notre période de réflexion se clôt alors qu'une nouvelle formation de paysagiste est finalement créée à Versailles, en $1976^{41}$; c'est l'actuelle École nationale supérieure du paysage où, durant plus de dix ans, la formation des ateliers s'est partagée entre deux filières, autour de M. Corajoud d'une part et de B. Lassus d'autre part. Ce dernier qui a contribué avec, entre autres, J. Sgard et P. Dauvergne, à l'épisode du CNERP, fermé en 1978, est à l'origine d'une formation de DEA en Paysage en 1991 à l'École d'architecture de Paris-la-Villette. 


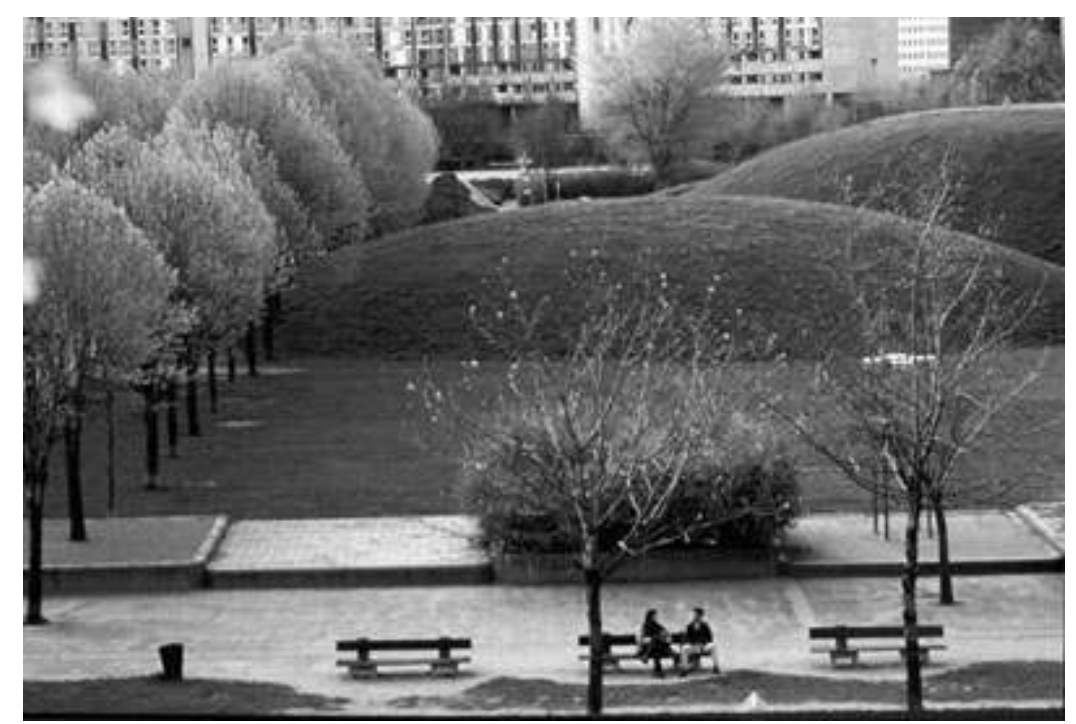

Villeneuve à Grenoble. Paysagistes : Corajoud, Ciriani, Huidobro (AUA) @ Gérard Dufresne.

Il nous semble que c'est bien dans le travail de ces «pionniers » que se sont réalisés le changement d'échelle des projets et la mise en place de modes d'intervention fondés sur les spécificités de la pratique paysagiste que sont l'observation du site, le dépassement des limites, l'interprétation du programme, la soumission aux forces du milieu vivant, la maitrise du substrat et modelage du sol, afin de mieux accueillir l'activité humaine.

Le primat du site et la question de la forme

Le rôle des espaces ouverts, non comme antidote à une expansion urbaine redoutée mais comme élément indissociable et structurant du développement urbain et de l'art de vivre en ville, s'est développé au cours du $\mathrm{xx}^{\mathrm{e}}$ siècle sans pour autant constituer un acquis définitif.

La connaissance du passé proche que nous avons exploré participe ainsi à la constitution de l'identité du paysagiste. Cet épisode nous semble important à éclairer en détail, car les réalisations témoignant de cette période sont peu nombreuses et représentent un patrimoine particulièrement fragile. Les décrire, comprendre leur genèse et leurs atouts, c'est aussi contribuer à leur préservation et fonder les pratiques de gestion et de restructuration en évitant les contresens.

58 Il conviendrait de poursuivre l'exploration des notions rencontrées, dans leur origine, leur contenu et leurs vecteurs de transmission depuis le tournant $\mathrm{du} \mathrm{xx}^{\mathrm{e}}$ siècle. C'est pourquoi la généalogie de la compétence paysagiste dans ses différentes composantes et disciplines ouvre une piste fructueuse. 


\section{NOTES}

1. Selon l'expression de J. Fourastié dans son célèbre ouvrage Les Trente Glorieuses ou la révolution invisible de 1946 à 1975, Paris, Pluriel-Fayard, 1979.

2. B. Blanchon, Pratiques paysagères en France de 1945 à 1975 dans les grands ensembles d'habitations, Rapport de recherche, Programme Cités projets, Plan Construction et Architecture, Ministère de l'Équipement, 1998.

3. B. Blanchon « Les paysagistes en France depuis 1945 ; l'amorce d'une indiscipline ou la naissance d'une profession ", in V. Picon-Lefebvre (dir.), Les espaces publics modernes, Paris, Le Moniteur, juin 1997, p. 121-210 ; avec M. Audouy, Pratiques paysagères en France au XXe siècle, Figures d'une évolution, Ministère de l'Environnement/ENSP, 1998 ; « Les paysagistes français de 1945 à 1975, l'ouverture des espaces urbains ", Les annales de la recherche urbaine, « Paysages en villes », $n^{\circ} 85$, janvier 2000, p. 20-29; « Paysagiste, naissance d'une profession ", in S. Texier (dir.), Les parcs et jardins dans l'urbanisme parisien $\mathrm{XIX}^{e}$-XXe siècles, Paris, Action artistique de la Ville de Paris, 2001, p. 258-266 ; Notices biographiques sur E. Redont, F. Duprat, A. Riousse, J.-C. Saint-Maurice, M. et I. Bourne, J. Simon, M. Corajoud et A. Chemetoff, in M. Racine (dir.), Créateurs de jardins et paysages $d u X_{X I}^{e}$ au XIXe siècle, tome 2, ENSP-Actes Sud, 2002 ; « Towards a Genealogy of Landscape competence ", in T. Andresen (dir.), From the National Stadium to the Gulbenkian Garden, Francisco Caldeira Cabral and the First Generation of Portuguese Landscape Architects (1940-1970), Lisbonne, Calouste Gulbenkian Fondation, 2003, p. 122-133.

4. De 1928 à 1959, réunions régulières d'architectes unis par la volonté de rompre avec le passé et la foi dans la technique ; moments culminants de militantisme et de formulation doctrinale.

5. Le sigle DPLG (diplômé par le gouvernement) suffit alors à les différencier des entrepreneurs et pépiniéristes.

6. F. Dubost, «Le traitement du site, logiques savantes et enjeux professionnels », in

R. Moulin (dir.), Sociologie de l'art, Paris, La Documentation française, 1986, p. 226.

7. F. Champy, « Les architectes, les urbanistes et les paysagistes », in T. Paquot et

al. (dir.), La ville et l'urbain, l'état des savoirs, Paris, La Découverte, 2000, p. 217.

8. Notamment ; É. Redont (1862-1942) ; H. Prost (1874-1959) ; J. Gréber (1882-1962) ;

J. C. Moreux (1889-1956).

9. Voir M. Racine (dir.), Créateurs de jardins et de paysages en France de la Renaissance au XXI siècle, t. 2, Arles, Versailles, Actes Sud/Ensp, 2002, p. 140-141.

10. J.-C.-N. Forestier, Grandes villes et systèmes de parcs, Paris, Hachette, 1906 (rééd. Norma Éditions, 1997).

11. Voir B. Leclerc (dir.), Jean-Claude-Nicolas Forestier, 1861-1930. Du jardin au paysage urbain, Actes du colloque international de 1990, Paris, Picard, 1994.

12. Voir J.-L. Cohen, A. Lortie, Des fortifs au périf, Paris, Picard-Pavillon de l'Arsenal, 1992.

13. Ce titre est reconnu par l'IFLA mais toujours contesté par l'Ordre des architectes. Il a pourtant été admis au concours international de la Villette. Voir F. Dubost, « Les paysagistes et l'invention du paysage ", Sociologie du travail, vol. 35, $\mathrm{n}^{\circ} 4,1983$, p. 438-439.

14. Voir note 8. 
15. L'Exposition internationale des arts décoratifs et industriels modernes de 1925 à Paris, où la section de l'Art des jardins est caractérisée par des jardins minéraux et géométriques, conçus le plus souvent par des architectes.

16. L'ordre ouvert caractérise les espaces organisés selon les principes de la Charte d'Athènes. Les bâtiments sont implantés par rapport à leur orientation vis-à-vis du soleil et libérés des contraintes du tracé des voiries. Voir J. Lucan, Architecture en France 1940-2000, Paris, Le Moniteur, 2001, p. 63.

17. T. Leveau, « Espaces verts de l'habitat », Urbanisme, vol. 24, n 39-40, 1955, p. $177-180$.

18. Office public d'habitations à bon marché du département de la Seine, créé en 1915. 19. Voir P. Dehan, B. Jullien, «Au détour du chemin de grue », in V. Picon-Lefebvre (dir.), Les espaces publics modernes, Paris, Le Moniteur, 1997, p. 33-55.

20. M. Bonilla, F. Toma, D. Vallat, L'immeuble collectif des années 50, Saint Étienne ; de la place Foch à Beaulieu, Publications de l'Université de Saint-Étienne, 1993, p. 128.

21. Ministre de la Reconstruction et de l'Urbanisme de septembre 1948 à juin 1953 et ministre de la Reconstruction et du Logement d'août à septembre 1954.

22. B. Barraqué, Le paysage et l'administration, Rapport de recherche, ministère de l'Urbanisme, du Logement et des Transports, direction de l'Urbanisme et des Paysages, Mission de la recherche urbaine, février 1985, p. 67-68.

23. Haie vive traditionnelle du centre de la France.

24. C'est-à-dire directement sur le terrain, sans intermédiaire, ni document graphique, ni mise au point ou forfait préalable, les coûts étant traités à prix réels des tâches exécutées.

25. Avec J.-B. Perrin et Met de Penningen, en 1967.

26. Voir A. Fourcaut, «Trois discours, une politique ? », Urbanisme, $n^{\circ} 322$, janv.-fév. 2002, p. 39-45.

27. En 1951, les besoins sont évalués à 320000 logements pendant trente ans alors qu'il s'en construit 70000 par an. L'objectif de 240000 logements est atteint en 1956. La loicadre du 7 août 1957 programme pour cinq ans les crédits nécessaires à la construction de 300000 logements par an. En 1975 on atteint 514000 logements.

28. Pour une présentation de ses travaux voir Urbanisme, $n^{\circ}$ 62-63, 1959.

29. P. Mas de 1948 à 1965, rejoint par J. Challet, L. Gendre, E. Mauret et P. Pelletier ; tous travaillent dans le champ de l'urbanisme.

30. Couvert dense de végétaux persistants ménageant la fraîcheur jusqu'au plus fort de l'été.

31. L'objectif de ne pas altérer le réseau hydrographique souterrain de la colline des Aygualades n'a pu être maintenu au final, les opérations voisines ne se souciant pas d'un tel objectif.

32. "Récréation et espaces verts aux Pays-Bas ", thèse de l'Institut d'urbanisme de Paris, sous la direction de J. Royer et du professeur Bijhouwer, 1958. Voir A. Vigny, Jacques Sgard, paysagiste et urbaniste, Liège, Mardaga, 1984.

33. Le paysage d'aménagement ou « Grand paysage » est la version française du landscape planning alors inconnu en France.

34. Notamment J. Challet qui effectue un stage au Danemark en 1947. Voir M. Racine (dir.), op. cit., p. 285.

35. I. Bourne-Cloppenburg, née en 1933, jeune Allemande d'origine hollandaise est auditrice libre à la Section du paysage après des études en Angleterre. Voir M. Racine (dir.), op. cit., p. 272-273. 
36. Le paysagiste J. Bernard est embauché par la Société centrale d'équipement du territoire (SCET), rattachée à la Caisse des dépôts et consignations.

37. Délégation à l'aménagement du territoire et à l'action régionale (DATAR), créée en 1963 ; Service d'études techniques des routes et autoroutes (SETRA). Les Organisations d'études et d'aménagement des aires métropolitaines (OREAM), notamment, créées de 1966 à 1972 par la DATAR autour de cinq des huit métropoles d'équilibre mises en place par la loi d'orientation foncière (LOF) de 1967, rassemblent des équipes pluridisciplinaires auxquelles participent les paysagistes J. Sgard, P. Dauvergne, M. Viollet.

38. Plan Urbain, 1989.

39. Corajoud, Ciriani, Huidobro, « paysagistes urbains » à l'Atelier d'architecture et d'urbanisme.

40. Voir B. Blanchon, Pratiques paysagères en France au $\mathrm{XX}^{e}$ siècle, Figures d'une évolution, p. 62-70, op. cit. note 3 .

41. La première promotion d'étudiants rentre en septembre 1975 devançant la signature du décret en 1976. Ce n'est qu'en 1995 que l'École nationale supérieure du paysage obtient son autonomie, l'École nationale supérieure d'horticulture s'installant alors à Angers.

\section{RÉSUMÉS}

Il s'agit ici d'éclairer la compréhension du rôle des paysagistes après la seconde guerre mondiale, notamment leur participation à la fabrication du paysage urbain dans les grands ensembles d'habitation. Cet épisode peu connu montre la production d'une génération de professionnels de l'aménagement, chargés de la conception des espaces ouverts urbains, actifs durant la période dite des Trente Glorieuses (1945-1975), porteurs d'un héritage lié à la pratique des parcs et jardins et confrontés à une nouvelle échelle de projet sous l'impulsion des mutations profondes de la société. L'examen de quelques réalisations permet d'identifier trois étapes dans leur contribution à la constitution d'un nouveau métier. Afin de caractériser la pratique paysagiste, ces différentes opérations sont d'abord décrites en suivant le fil directeur du rapport au site. Nous chercherons en outre, à examiner la place accordée au paysagiste dans l'interprétation de la commande et dans la définition des espaces ouverts par l'implantation des bâtiments, ainsi qu'à cerner la posture du paysagiste dans le dessin des espaces à partir du matériau végétal, du travail du sol ou de la notion de temps.

Bernadette Blanchon-Caillot, Landscape-architects' skills and practices in social mass housing, 1945-1975

The purpose of this article is to understand the role of the post-war landscape architects (after World War 2), highlighting their contribution to creating urban landscape in social mass housing (grands ensembles). This somewhat unknown episode illustrates the production of a generation of city planning professionals in charge of conceiving urban open spaces during the so-called Trente Glorieuses (1945-1975). This generation was responsible for the legacy of parks and gardens and was faced with a new scale of project as a result of important changes in society. Looking at a selection of their works, three steps can be identified in their contribution to the 
consolidation of a new profession. In order to characterize the practice of landscape architecture, these examples are described according to the thread of their relationship with the existing site. We consider the place given to the landscape architect in the interpretation of the original demand and in the definition of open spaces according to how the buildings are set up. Then we position the designer and his conception of open spaces according to vegetation, soil and the dimension of time.

\section{INDEX}

Mots-clés : paysage urbain, grands ensembles, espaces verts, pratiques paysagistes, lecture de projet, échelles

Keywords : urban landscape, site, social mass housing, landscape architecture practises, deciphering projects, green spaces, scales

\section{AUTEUR}

\section{BERNADETTE BLANCHON-CAILLOT}

Architecte dplg, Maître de conférences à l'École nationale supérieure du paysage, Département des sciences de l'homme et de la société, Le potager du roi - 10, rue du Maréchal-Joffre,78009 Versailles Cedex, b.blanchon@versailles.ecole-paysage.fr 